\title{
On articulated plates with micro-slits to tackle low-frequency noise
}

\author{
Massimo Emiliano D'Elia* (D), Thomas Humbert (D), and Yves Aurégan (D) \\ Laboratoire d'Acoustique de l'Université du Mans, LAUM-UMR CNRS 6613, Avenue Olivier Messiaen, 72085 Le Mans Cedex 9, \\ France
}

Received 13 April 2021, Accepted 21 June 2021

\begin{abstract}
In recent years, new concepts of acoustic absorbers dedicated to the reduction of low-frequency noise have been developed. Among them, liners with moving parts, such as membrane-based liners, have been an object of particular interest. In the present paper, we propose a liner concept based on a cantilever beam made of articulated plates with micro-slits. Compared to membrane technologies, these micro-slits introduce a small leakage from the backing cavity that reduces the high compressibility effects occurring at very low frequencies in a small cavity. An acoustic liner including an ensemble of such articulated plates has been fabricated and characterized for grazing acoustic incidence in absence and in presence of flow. Measurements in an impedance tube at normal incidence have also been performed, and perfect absorption is obtained at a frequency where the liner thickness corresponds to $1 / 16$ th of the acoustic wavelength. A new and simple model is proposed to predict the attenuation of this type of acoustic treatment. The results are in good agreement with the measurements, indicating a correct identification of the physical phenomena here at stake.
\end{abstract}

Keywords: Acoustic materials, Metamaterials, Vibrating beams

\section{Introduction}

Reducing low-frequency sound with small size devices remains challenging. As a result, various new sound absorber concepts for the reduction of low-frequency noise have been developed in recent years. Conventional locally reacting absorbers consist of a honeycomb structure acting as a cavity and a perforated or micro-perforated plate where the air in the holes acts as a resistance and also as an oscillating mass $[1,2]$. In this case, the resonance frequency is somewhere between the quarter-wave resonance of the cavity ( $f=c_{0} / 4 \mathrm{~B}$ where $c_{0}$ is the speed of sound and $B$ is the height of the cavity) and the Helmholtz resonance frequency $\left(f=c_{0} / 2 \pi \sqrt{\sigma / B e}\right.$ where $\sigma$ is the ratio of the area of the holes to the total area (open area ratio) and $e$ is the effective thickness of the perforations). To reduce the height $B$, several strategies have been adopted [3-5], like space-coiling structures [6-9], slow-sound materials [10, 11] or by increasing the effective thickness of the plate [12]. These new structures lead to perforated plates with a low open area ratio $\sigma$ in order to be able to reduce the height $B$. As the velocity in the holes is equal to the incident acoustic velocity divided by $\sigma$, non-linear effects can easily occur in the holes when the amplitude of the incident wave becomes sufficiently large. In many engineering applications, grazing flow is present and its effect on the impedance of the material is inversely proportional to $\sigma$
$[1,11]$. Therefore, in the case of high sound levels or in the presence of flow, the additional constraint of having a high open area ratio is also required in the design of these new materials.

In this regard, the use of elastic membranes and decorated elastic membranes as sound absorbers is an interesting option [13-16]. In this case, the membrane can represent a large part of the total surface area and the frequency can easily be reduced by increasing the added mass [17]. The effect of a grazing flow has already been studied on this type of structure [18, 19], however it has the disadvantage of containing an encapsulated volume of air which can expand in the presence of a depression causing static deformation of the membrane. This is why other vibrating structures have been considered such as cantilever beams but with micro slits around them [20-22].

The present paper deals with the concept of cantilevered thin blades that can vibrate above a cavity. In order to soften these blades, two I-shaped cuts are introduced. The first one makes the fixation less rigid while the second, realized in the middle of the beam, leads to bi-articulated blades with two degrees of freedom and a low stiffness.

First, a simple analytical model is proposed to predict the behavior of the blade. Then, a parametric study is performed in order to design experimental samples. The first experiments are conducted in an impedance tube, where both the acoustic in the duct and the vibration of the beam

*Corresponding author: massimo_emiliano.delia.etu@univ-lemans.fr 
are measured. The closeness of the experimental and the analytical results indicates that the right physical phenomena have been identified. Finally, a last set of experiments performed in a grazing flow facility gives hint about the possible application of such acoustic treatments to aircraft noise reduction.

\section{Analytical model}

\subsection{Model of a mono-articulated beam}

In order to predict the low-frequency behavior of the articulated beam with micro-slits, a simple model with a single degree of freedom is firstly considered. This model will be further referred as the mono-articulated one in the text, while a second structural mode will be introduced in Section 2.5 to constitute a bi-articulated beam model. In the case of the mono-articulated model depicted in Figure 1, the beam is assimilated to a plate rotating around its end with a restoring torque due to the deformation. Considering a time dependance $\exp (j \omega t)$ where $\omega$ is the frequency, the motion equation that links the rotation angle $\theta$ to the pressure difference between the two faces of the beam $\Delta p$ reads in the frequency domain:

$$
-J \omega^{2} \theta=\sum M=l \Delta p S_{b} / 2-\beta \theta-\gamma j \omega \theta .
$$

The moment of inertia $J$ is equal to $J=\rho_{b} e S_{b} l^{2} / 3$, where $\rho_{b}$ is the density of the beam, $e$ its thickness, $S_{b}$ its area and $l$ its length. The two coefficients $\beta$ and $\gamma$ are linked respectively to the stiffness and to the damping of the beam.

After having defined the mean normal velocity of the beam $v_{b}=j \omega l \theta / 2$, equation (1) can be transformed into an equation that links the pressure difference $\Delta p=p_{i}-p_{c}\left(p_{i}\right.$ and $p_{c}$ are the pressure just above and just under the beam in the cavity, respectively) to $v_{b}$ :

$$
\begin{aligned}
\Delta p & =\left[j \omega \frac{4}{3} \rho_{b} e\left(1-\frac{\omega_{b}^{2}}{\omega^{2}}\right)+\rho_{0} c_{0} \delta\right] v_{b} \\
& =\rho_{0} c_{0}\left[j k_{b} e_{b} \frac{\omega}{\omega_{b}}\left(1-\frac{\omega_{b}^{2}}{\omega^{2}}\right)+\delta\right] v_{b}=\rho_{0} c_{0} Z_{b} v_{b},
\end{aligned}
$$

where $\omega_{b}$ is the resonance frequency of the beam, $k_{b}=\omega_{b} / c_{0}, e_{b}=4 \rho_{b} e /\left(3 \rho_{0}\right)$ is an equivalent thickness of the beam, $\delta$ is the dimensionless structural damping, $\rho_{0}$ is the fluid density and $c_{0}$ is the speed of sound.

In the slits, the same pressure difference $\Delta p$ induces a mean velocity $v_{s}$ so that

$$
\begin{aligned}
\Delta p & =\rho_{0} c_{0}\left(R_{s}+j k_{b} e_{s} \omega / \omega_{b}\right) v_{s} \\
& =\rho_{0} c_{0} Z_{s} v_{s},
\end{aligned}
$$

where $R_{\mathrm{S}}$ is the resistance of the slit, $e_{\mathrm{s}}$ its equivalent length and $Z_{\mathrm{s}}$ its specific impedance. Then, the continuity of the acoustic mass flux implies that

$$
S_{i} v_{i}=S_{b} v_{b}+S_{s} v_{s}=S_{c} v_{c},
$$

where $S_{i}$ is the area corresponding to one periodic cell of the material, $v_{i}$ the incident vertical mean velocity on one cell, $S s$ the slits area, $S_{c}$ the transverse area of the cavity and $v_{c}$ the mean vertical velocity at the entrance of the

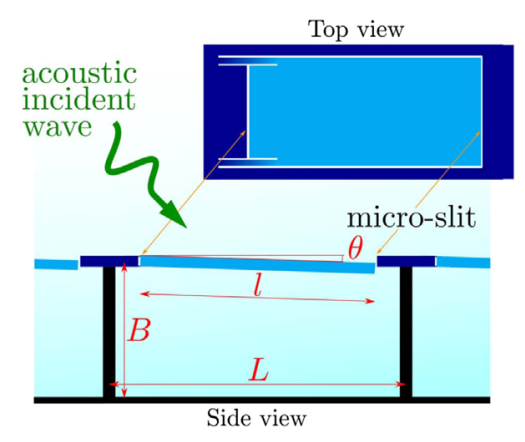

Figure 1. Sketch of a mono-articulated blade of length $l$ with laser cut micro-slits. The structure is attached to a cavity with a thickness of $B$ and a length of $L$. The rotation angle of the beam is denoted $\theta$.

cavity. The impedance of the cavity $Z_{c}$ is introduced by writing

$$
p_{c}=\rho_{0} c_{0} Z_{c} v_{c}=\frac{\rho_{0} c_{0}}{j \tan \left(k_{b} B \omega / \omega_{b}\right)} v_{c}
$$

where $B$ is the thickness of the cavity. The continuity equation (3) can then be written as

$$
\left[\frac{S_{b} / S_{i}}{\rho_{0} c_{0} Z_{b}}+\frac{S_{s} / S_{i}}{\rho_{0} c_{0} Z_{s}}\right]\left(p_{i}-p_{c}\right)=v_{i},
$$

from which the impedance $Z_{\mathrm{i}}$ seen by an incoming wave can be calculated:

$$
Z_{i}=\frac{p_{i}}{\rho_{0} c_{0} v_{i}}=\left[\frac{S_{b} / S_{i}}{Z_{b}}+\frac{S_{s} / S_{i}}{Z_{s}}\right]^{-1}+\frac{S_{i}}{S_{c}} Z_{c} .
$$

This impedance is that of a resonator placed in parallel with a resistor and loaded by a cavity.

\subsection{Acoustical effects of the micro-slits}

In order to obtain low resonance frequencies, a cantilever and two I-shaped cuts were made in the blade using a very thin laser beam. The used technology creates a slightly conical groove with a width of $50 \mu \mathrm{m}$ at the narrowest point. In the slit, since the dimensions are much smaller than the studied wavelengths, the flow can be considered as incompressible. In this case, the pressure difference $\Delta p$ between the two faces of the beam is linked to the mean velocity in the slit $v_{s}$ by [23]:

$$
\frac{\Delta p}{\rho_{0} c_{0} v_{s}}=-\frac{j \omega e}{c_{0}}\left[1-\frac{\tanh (\kappa s)}{\kappa s}\right]^{-1}
$$

where $s$ is the width of the slit opening. Here, $\kappa=\sqrt{j \omega \rho_{0} / 4 \mu}=(1+\mathrm{j}) / 2 \delta_{v}$, where $\delta_{v}=\sqrt{2 \mu / \omega \rho_{0}}$ is the viscous boundary layer thickness and $\mu$ the dynamic viscosity of air. This result comes directly from the application of Newton's law, where the velocity time derivative is linked to the pressure difference $\Delta p$ between the two sides of the slits and to the spatial second derivative of the velocity in the longitudinal direction, i.e. $\rho_{0} \partial v / \partial$ $t=-\Delta p+\mu \partial^{2} v / \partial y^{2}$. 
When the ratio $s / \delta_{v}$ is small, a Taylor expansion permits to approximate the resistance by $R_{s}=(12 \mu e) /$ $\left(\rho_{0} c_{0} s^{2}\right)$, while the imaginary part reads $(6 \omega e) /\left(5 c_{0}\right)$. Thus, the impedance can be written as $Z_{s}=R_{s}+j\left(\omega / \omega_{b}\right) e_{s}$ where $R_{s}$ and $e_{s}=6 / 5 e k_{b}$ are independent of the frequency. The above values only take into account what happens in the slit without considering what happens when air enters or exits the slit. Usually, these effects are accounted for by introducing an extra length that is not considered here [2].

\subsection{Analytical results}

The predicted acoustic behaviour of the system is displayed in Figure 2. The absorption at normal incidence $\left(\alpha=1-\left|\left(1-Z_{i}\right) /\left(1+Z_{i}\right)\right|^{2}\right)$ as well as the imaginary and real parts of the impedance $Z_{i}$ are shown as functions of the frequency normalised by the beam resonance frequency. The values of the parameters given in the figure caption correspond to values close to those obtained experimentally. It can be seen that perfect absorption at normal incidence can be achieved at a frequency that is closed to the resonance frequency of the beam. What generally limits the possibility to obtain low frequency attenuation with liners is the presence of the backing cavity whose reactance (green curve on Fig. 2) goes towards $-\infty$ as $1 / k_{0} B$. The beam creates an additional reactance that is negative at low frequency and positive at high frequencies, with a rapid variation at its resonance frequency where zero is reached. If the beam reactance rises high enough, the cavity reactance can be counterbalanced and a total zero reactance is obtained for a frequency close to that of resonance. In the particular case displayed in Figure 2, the ratio between the wavelength at the beam resonance and the thickness of the cavity is 16.2 .

At the same time, in order to obtain perfect absorption, which occurs when $Z_{i}=1$ for normal incidence, the coupled system resistance must be equal to 1 . The resistance comes mainly from the dissipation in the micro-slits. However, near the resonance frequency the slits are short-circuited, and the velocity in the cavity comes mainly from the motion of the blade. As the structural damping of the beam is considered to be significantly lower than the slit resistance, the short-circuit in the vicinity of the resonance produces a localised dip in the resistance curve whose minimum value depends on the beam structural damping $\delta$. Then, an appropriate choice of $R$ and $\delta$ permits to obtain a resistance of 1 for the frequency at which the reactance is zero. Thus, perfect absorption is provided for a normally incident wave.

\subsection{Influence of the parameters}

The analysis of the effects of the problem parameters is illustrated in Figure 3 where, starting from the reference configuration already shown in Figure 2, the parameters are varied one by one.

The first parameter that can be changed is the mass of the beam, see Figure 3a. In practice, this mass can be changed either by modifying the material and thus the density, or by changing the thickness of the beam. Obviously, these
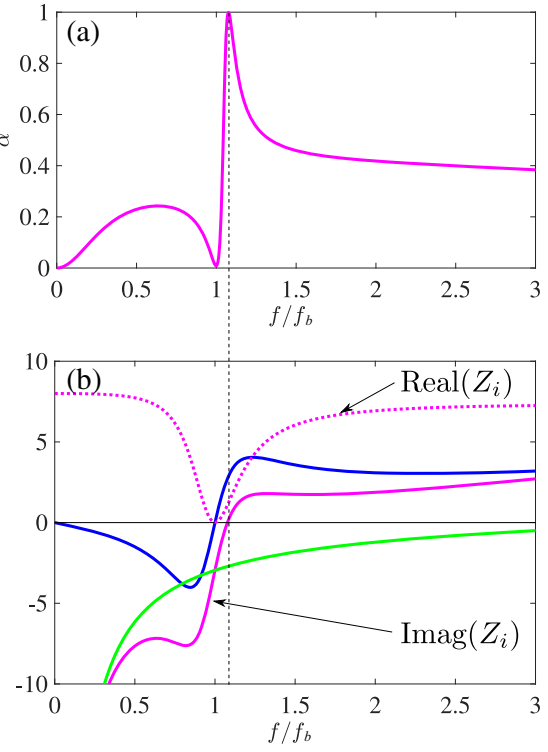

Figure 2. (a) Absorption coefficient and (b) impedance computed from the mono-articulated model as a function of the frequency normalised by the beam resonance frequency. $S_{b} / S_{i}=$ $0.48, S_{s} / S_{i}=0.0083, S_{c} / S_{i}=0.825, k_{b} e_{b}=0.8133, \delta=0.01$, $R=S_{i} / S_{s} R_{s}=8, k_{b} e_{s}=0.0065, k_{b} B=0.39$. The green curve is the imaginary part induced by the cavity and the blue curve is the imaginary part induced by the beam and the micro-slits.

changes will also involve a change in the beam resonance frequency $f_{b}$. A reduction in mass $\left(k_{b} e_{b}\right.$ decreases) leads to a softer resonance but with the same amplitude. This leads to an increase in the frequency where the system is efficient, as well as a widening of the absorption bandwidth. A small adjustment of the structural damping $\delta$ is needed to achieve perfect absorption again.

The second parameter that could be modified is the thickness of the cavity $B$, see Figure 3b. As it is decreased, the zero crossing of the reactance moves to higher frequencies. At these frequencies, the corresponding resistance doesn't display a dip and is thus too high to induce good absorption.

The third parameter that is studied is the structural damping $\delta$, see Figure 3c. The increase of $\delta$ makes the resonance less pronounced and increases the overall resistance (the resistance dip is less marked). On the other hand, it can be noted that the very low absorption values that were reached when $f=f_{b}$ are now increased because the structural damping takes over the damping in the slits when $\delta$ is large enough.

The last parametric study considers the resistance in the slits and the results are shown in Figure 3d. For this, we consider the resistance $R=S_{i} / S_{s} R_{s}$, which takes into account the percentage of open surface. Therefore, it is referred to the total surface. If the resistance increases, the width of the system efficiency peak is reduced. When $R=1$, the system works very differently. Indeed, the change in reactance due to the beam resonance is very weak and the reactance passes through zero for higher frequencies $(f / f b=1.84$ in this particular case). Such a frequency is outside the resistance dip and the resistance value is then 

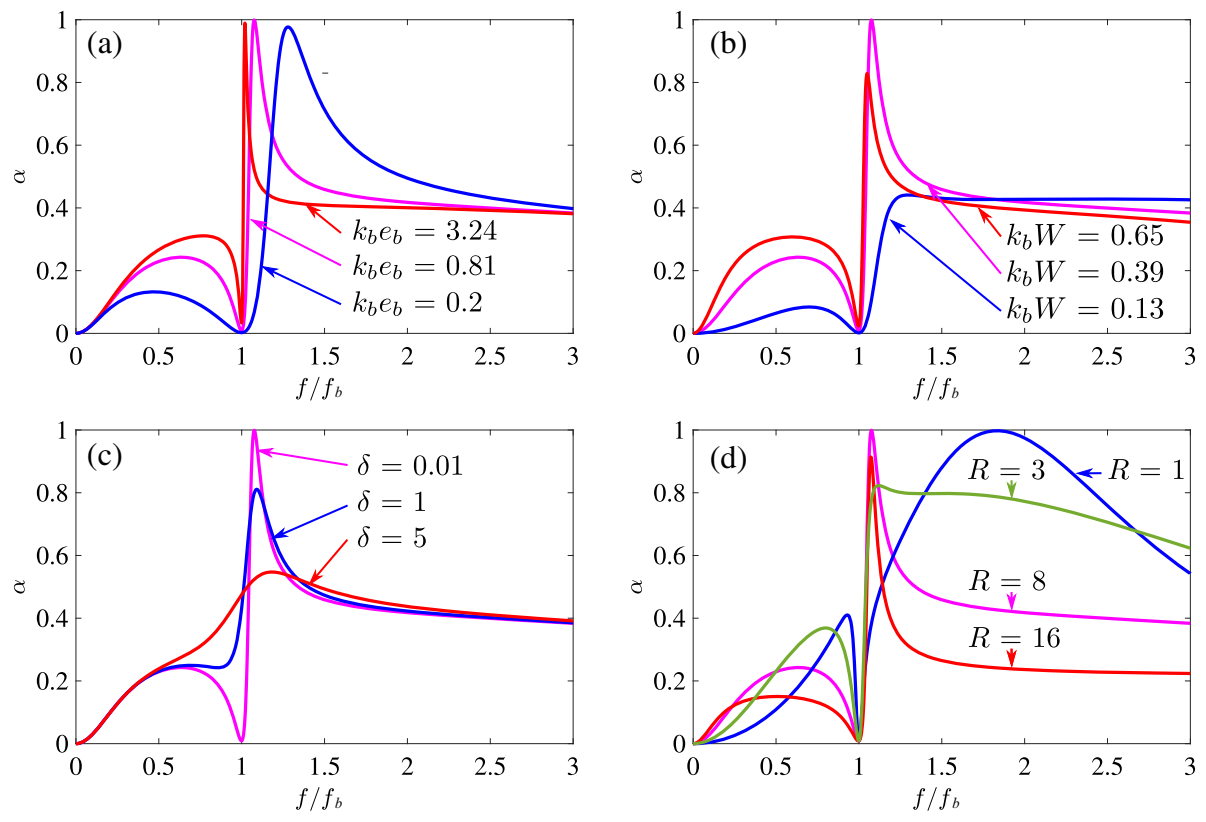

Figure 3. Absorption coefficient computed by the mono-articulated model as a function of the normalised frequency. (a) Variation of the mass of the beam. (b) Variation of the thickness of the cavity. (c) Variation of the structural damping. (d) Variation of the resistance. The magenta curves are the same that in Figure 2a.

very close to the nominal value of 1 , which leads to perfect absorption for a frequency close to twice the resonance frequency of the beam. In fact, in this operating mode, the beam is in its inertial regime. Perfect absorption is then obtained with a system that consists of a mass (the beam), a resistance (the slits) placed in parallel and a stiffness (the cavity). Such systems are called In-Parallel Resonator and have been investigated in detail in [22]. In the present paper, we are not interested in this type of operating mode, since the goal was to take an acoustic advantage of the beam resonance that occurs at lower frequencies. Finally, an interesting behaviour appears for an intermediate resistance $(R=3)$ value, where both modes work simultaneously. In this case, the operating band of the absorber is widened, in counterpart of a decrease in maximal absorption which is now of the order of $\alpha=0.8$ for $1.05<f$ / $f_{b}<1.85$.

\subsection{Influence of other modes of the plate}

In any deformable system, several modes can appear. The displacement of the beam is then written as a sum of the displacements induced by each of these modes. Thus, the continuity equation (3) now reads

$$
S_{i} v_{i}=\sum_{j} S_{b} v_{b j}+S_{s} v_{s}=S_{c} v_{c}
$$

where $v_{b j}$ is the mean velocity induced by the $j$ th mode. Then, the effect of the various modes $j$ can be modelled by writting the impedance of the $j$ th mode as:

$$
Z_{b j}=\mathrm{j} k_{b} e_{b j} \frac{\omega}{\omega_{b}}\left(1-\frac{\omega_{b j}^{2}}{\omega^{2}}\right)+\delta_{j} .
$$

Considering that this impedance is in parallel with that of the micro-slits and in series with the one of the cavity leads to the following expression for the impedance of the coupled system:

$$
Z_{i}=\left[\sum_{j} \frac{S_{b} / S_{i}}{Z_{b j}}+\frac{S_{s} / S_{i}}{Z_{s}}\right]^{-1}+\frac{S_{i}}{S_{c}} Z_{c} .
$$

The three parameters that define each of the modes (equivalent mass, resonance frequency and modal damping) can be determined either analytically from the beam deformation equation, numerically or experimentally.

To examine the effect of the additional modes, two modes are used to calculate the impedance and the absorption displayed in blue and compared in Figure 4 to the results with only one mode. It can be observed that the first absorption peak is only slightly modified and that the second peak appears near the resonance frequency of the second mode. The resistance remains close to its nominal value (here $R=8$ ) except near the resonant frequencies where dips are found. An interesting effect of the presence of the additional mode is that the reactance decreases just after the first peak. Between peaks the reactance therefore remains close to zero, which is usually required for large acoustic attenuation.

\section{Experimental validation}

To test the validity of the above model, measurements were performed on a $0.5 \mathrm{~mm}$ thick composite beam made with a carbon fibre/epoxy material (M21E/IMA). The density of this material was measured to be $1550 \mathrm{~kg} \mathrm{~m}^{-3}$. The 

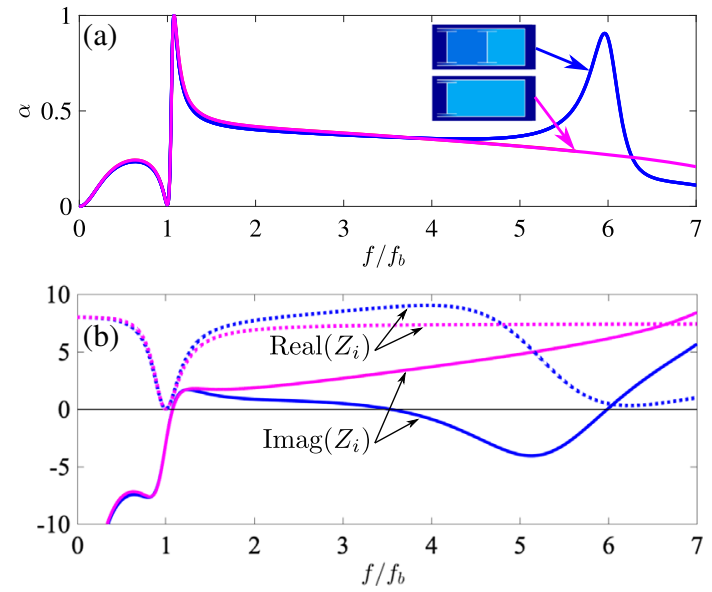

Figure 4. (a) Absorption coefficient and (b) impedance as a function of the normalized frequency when one (mono-articulated beam, in magenta) or two (bi-articulated beam, in blue) modes are considered. For the first mode, the parameters are the same that in Figure 2. The second mode is defined by $\omega_{\mathrm{b} 2}=6.05 \omega_{b}, k_{b 2} e_{b 2}=k_{b} e_{b} / 8$ and $\delta_{2}=2.5 \delta$.

Poisson's ratio (which does not have a great influence on the vibration of the beam) is estimated to be 0.35 . The Young's modulus was adjusted to a value of $75 \mathrm{GPa}$ so that the first calculated resonance frequency corresponded to the measured value.

Two samples have been made: one for measurements in a normal incidence tube (cut in a disk of diameter $38 \mathrm{~mm}$ ) and a second one for measurements in the wall of a rectangular duct (cut in a rectangular plate of $120 \times 50 \mathrm{~mm}^{2}$ ). For these two samples, the micro-cutting was performed in the same way and with the same geometry. The micro-slits were made by laser micro-cutting with high precision machining. The resulting micro-slits are $50 \mu \mathrm{m}$ wide (see inset pictures of Fig. 6).

Using the geometry and the material parameters and neglecting air loading on the beam, the vibration modes of the beam can be computed using a finite element method (COMSOL). The first two vibration modes are shown in Figure 5 . The first one $(f=709 \mathrm{~Hz}$, see Fig. 5a) corresponds to an almost pure rotation of the whole beam. The stiffness results from the deformation of the two arms at the base of the beam. The second mode $(f=4412 \mathrm{~Hz}$, see Fig. $5 \mathrm{~b})$ is a bending mode while the third mode $(f=4453 \mathrm{~Hz})$ is a torsion mode which has an average velocity equal to 0 . Therefore, it is assumed not to interfere with the acoustics.

\subsection{Normal incidence measurements}

The circular sample is glued onto a ring (inner diameter $30 \mathrm{~mm}$, outer diameter $38 \mathrm{~mm}$ ) and placed in an impedance tube which is made of steel tubes with an inner diameter of $30 \mathrm{~mm}$ and a wall thickness of $4 \mathrm{~mm}$, see Figure 6 . Four microphones (B\&K 4136 and 2670 with amplifier Nexus 2690) are used for the overdetermined separation of incident and reflected waves [24, 25]. The microphones are distant of $L_{2}=30 \mathrm{~mm}, L_{3}=100 \mathrm{~mm}$ and $L_{4}=285 \mathrm{~mm}$ in order to cover a large range of frequencies. The length

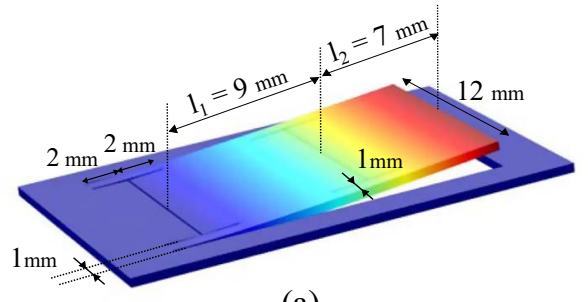

(a)

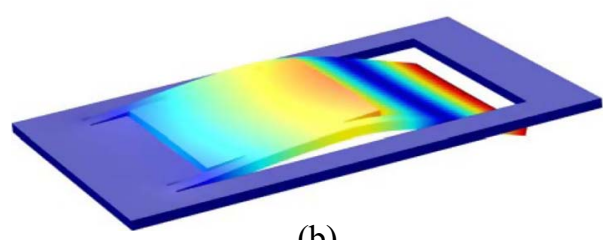

(b)

Figure 5. (a) First $(f=709 \mathrm{~Hz})$ and (b) second $(f=4412 \mathrm{~Hz})$ mode of the cantilever beam with the I-cut shaped slits, as computed using a finite elements method (Comsol). $l_{1}$ and $l_{2}$ are the lengths of the two parts of the beam.

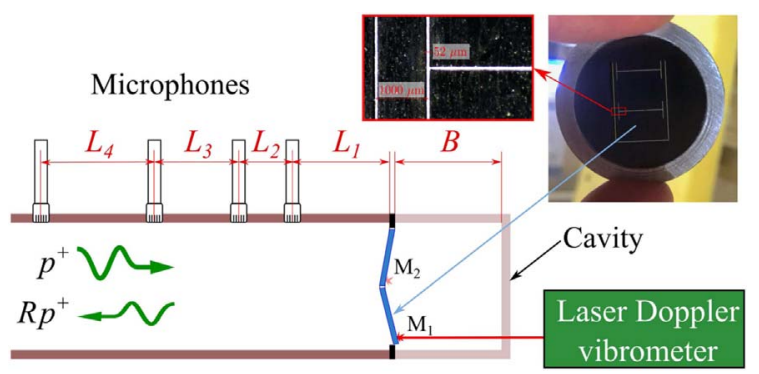

Figure 6. Schematic diagram of the setup for impedance tube experiments. Acoustic measurements are performed using four microphones separated by distances $\left.L_{1 \ldots 4}\right)$. Vibrations are evaluated using a Laser Doppler vibrometer, and two measurement points are defined on the beam. The back cavity of length $B$ can be removed to characterize the blade alone. The inset pictures display the composite sample used for the normal incidence measurements, as well as a zoom on the laser cut slits.

between the first microphone and the sample is $L_{1}=230 \mathrm{~mm}$. In order to measure the velocity induced by the beam vibration, a laser vibrometer impinges on the beam. After an in-situ calibration of the microphones, the signals from the microphones and from the laser vibrometer are transferred to a data acquisition system. The measurements are performed using a sine sweep excitation signal going from 100 to $4000 \mathrm{~Hz}$ with a step of $5 \mathrm{~Hz}$. At each frequency, all transfer functions are averaged over 500 cycles.

The measurement system allows to control the acoustic level of the incident wave. Several levels ranging from $110 \mathrm{~dB}$ to $140 \mathrm{~dB}$ were tested but no non-linear effects were detected.

\subsubsection{Vibrometer measurements}

First, the velocity of the bi-articulated beam when placed at the end of the tube (without cavity) was measured using a laser vibrometer, when acoustic excitation is 
present. From the sound pressures measured on the three microphones, it is possible to calculate the sound pressure $p_{i}$ that is applied to the plate. The velocities $v_{M_{1}}$ and $v_{M_{2}}$ measured respectively at the end and in the middle of the beam (i.e. at the end of the first plate composing the beam, see Fig. 6 where the points $M_{1}$ and $M_{2}$ are indicated) related to the incident pressure are plotted in Figure 7 . The first two modes of the bi-articulated plate can be seen very clearly. The first mode is at a frequency of $710 \mathrm{~Hz}$. For this mode, we find that $v_{M_{1}} /\left(l_{1}+l_{2}\right)=v_{M_{2}} / l_{2}\left(l_{1}\right.$ and $l_{2}$ are defined in Fig. 5). It indicates that this mode is very close to a rotation of the plate around its base articulation, without deformation. For the second mode $(f=3650 \mathrm{~Hz})$, the velocities are almost opposite $v_{M_{1}} \simeq-v_{M_{2}}$, which indicates that we are dealing with a bending mode as shown in Figure 5. An average velocity can be computed, assuming two rigid plates pivoted to each other, by $v_{b}=v_{M_{2}} / 2+$ $l_{1} v_{M_{1}} /\left(l_{1}+l_{2}\right)$. This averaged velocity is also plotted in Figure 7. Assuming a low radiation impedance of the tube and therefore zero sound pressure on the outside of the plate, this average velocity relative to the sound pressure on the plate is the inverse of the impedance of the beam defined by equation (2): $Z_{b}=p_{i} /\left(\rho_{0} c_{0} v_{b}\right)$.

These velocity measurements confirm that the impedance $Z_{b}$ can be described by the contribution of the first two modes with:

$$
\frac{1}{Z_{b}}=\frac{1}{Z_{b_{1}}}+\frac{1}{Z_{b_{2}}}
$$

where

$$
\begin{aligned}
& Z_{b_{1}}=j A_{1} \frac{f}{f_{1}}\left(1-\left(\frac{f_{1}}{f}\right)^{2}\right)+\delta_{1}, \\
& Z_{b_{2}}=j A_{2} \frac{f}{f_{2}}\left(1-\left(\frac{f_{2}}{f}\right)^{2}\right)+\delta_{2} .
\end{aligned}
$$

Thus, this vibration measurement allows to experimentally determine some parameters of the model described in Section 2.

Indeed, if some of them are easily computable such as the equivalent mass of the beam for the first mode, others are more difficult to evaluate. From eq. (2), the equivalent mass is computed from $A_{1}=k_{b} e_{b}=4 \rho_{b} \omega_{b} e /\left(3 \rho_{0} c_{0}\right)=10.55$. Note that the equivalent thickness of the beam is $e_{b}=0.81 \mathrm{~m}$. It corresponds to the thickness of air that would have to be set in motion to obtain the same effect. This shows the interest of making vibrate a solid part when we want to perform low frequency attenuation. If we simply consider that the beam is a rotating plate and that the stiffness comes from the deformation of the two arms of width $b=1 \mathrm{~mm}$ and length $a=4 \mathrm{~mm}$ at the base of the plate, the resonance frequency reads $\omega_{1}^{2}=0.5\left(E / \rho_{b}\right) b e^{2} /\left(B a l^{3}\right)$ where $E$ is the Young modulus, $B$ is the width of the plate and $l=l_{1}+l_{2}$ is its length. Using the dimensions and characteristics of the cut composite plate, the predicted frequency is equal to $f_{1}=1080 \mathrm{~Hz}$ while the measured

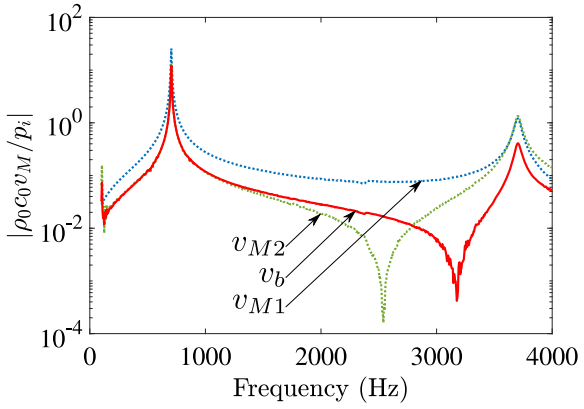

Figure 7. Dimensionless ratio between the velocities $v_{M_{1}}$ and $v_{M_{2}}$ and the pressure on the plate $p_{i}$. $v_{b}$ is the average velocity along the beam.

frequency is $f_{1}=710 \mathrm{~Hz}$. This difference comes from the crudeness of the model used to predict stiffness. Nevertheless, this model allows us to identify the main parameters influencing this resonance frequency and to compare it with that of a cantilever beam of the same dimensions without I-cuts, which is here given by $\omega^{2}=1.02\left(E / \rho_{b}\right) \mathrm{e}^{2} / l^{4}$, leading to a frequency $f=2680 \mathrm{~Hz}$. The structural damping is more complex to evaluate and is deduced from the vibrometer measurements: $\delta_{1}=0.01$.

The values of the three parameters of the second mode are deduced directly from the vibrometer measurements: $\delta_{2}=1.25, A_{2}=150, f_{2}=3730 \mathrm{~Hz}$. The measured impedance $Z_{b}$ (in blue) and the calculated impedance (in red) are shown in Figure 8. A correct agreement between these two values is found, especially in the vicinity of resonances where the imaginary part of $Z_{b}$ becomes zero.

\subsubsection{Acoustic measurements}

Using the four microphones described in Figure 6, the reflection coefficient $r$ can be obtained by an overdetermined separation of incident and reflected waves by means of a least-squares method. From $r$, the absorption coefficient $\alpha=1-|r|^{2}$ and the impedance $Z=(1+r) /$ $(1-r)$ can be easily computed. Here, the bi-articulated beam is backed up by a $30 \mathrm{~mm}$ deep closed cavity whose cross-section is as large as the incident tube (diameter $30 \mathrm{~mm})$.

To predict the impedance of this device, equation (5) is used. In this equation, the beam impedance $Z_{b}$ is deduced from the vibration measurements described in the previous subsection. The cavity impedance $Z_{c}$ can be computed from equation (4) with $B=30 \mathrm{~mm}$. The most tricky part to estimate is the acoustic impedance of the slit $Z_{s}$. Indeed, the slit resistance is extremely sensitive to the width of the slit $s$. If we use the relation $R_{s}=12 \mu \mathrm{e} /\left(\rho_{0} c_{0} s^{2}\right)$ we see that this resistance is inversely proportional to the square of $s$. In addition if one relates this impedance to the incident surface $R=S_{i}$ / $S_{s} R_{s}$, where $S_{i}=l_{s} s$ and $l_{s}=88 \mathrm{~mm}$ the total length of the slits, we see that the resistance $R$ is proportional to $s^{-3}$ ). The machining process of these micro-slits results in a slight conicity of the slit which therefore does not have a constant width $s$. On the height of $e=500 \mu \mathrm{m}$, it is estimated that 


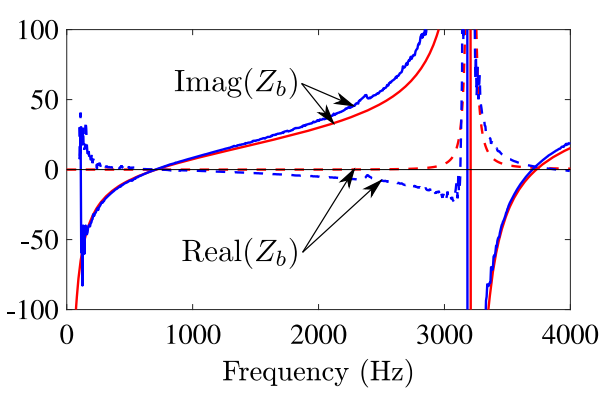

Figure 8. Impedance of the beam $Z_{b}$ deduced from the laser vibrometer measurements. The blue curves are the measurements and the red ones correspond to the fit described in the text.

the width goes from $50 \mu \mathrm{m}$ (see the photo under the microscope on Fig. 6) at the narrowest to $100 \mu \mathrm{m}$ on the other side of the plate. For a constant width $s=50 \mu \mathrm{m}$ the resistance is $R=16$ while for $s=100 \mu \mathrm{m}$ the resistance is $R=2$. It is therefore difficult to say more than $2<R<16$ and the exact value of $R$ had to be experimentally adjusted.

The measured absorption coefficient $\alpha$ and impedance $Z$ are plotted with a wide blue line in Figure 9. What happens on these curves in the vicinity of the first resonance frequency of the beam is very similar to what the model predicts. In particular, we can see that the maximum absorption frequency $(735 \mathrm{~Hz})$ is slightly higher than the first resonance frequency of the beam $(710 \mathrm{~Hz})$ which in fact corresponds to a very low absorption. We also note that the real part of the impedance (dashed line in Fig. 9b) tends at low frequency towards a constant which is the resistance $R$. We can therefore estimate the value of the resistance: $R=8.5$.

At this stage, all the parameters that describe the measured device are known and the impedance and absorption coefficient can be calculated (thin red curve on Fig. 9). This suggests that the proposed model takes into account the main effects that occur in such a device and that it is possible to tailor such a system for specific uses.

\subsection{Grazing incidence measurements}

In order to perform measurements with grazing incidence and grazing flow, a second sample was made from the same composite material plate and with exactly the same micro-cutting geometry. This sample is made in a plate of $120 \times 50 \mathrm{~mm}^{2}$, where 3 rows of 5 beams have been micro-cut, in blue on Figure 10. This plate was glued on a support (in black in Fig. 10) with 15 cavities of section $15 \times 22 \mathrm{~mm}^{2}$ and height $B=30 \mathrm{~mm}$.

During the measurements, this sample of 15 beams is flush mounted on the wall of a waveguide with a rectangular section. The height of the channel is $H=40 \mathrm{~mm}$ while the transverse dimension is $50 \mathrm{~mm}$. This means that the sample covers the entire width of the channel. This duct facility has already been introduced and described in [26, 27]. The acoustic waves are generated by two compression chambers which can be placed either upstream or either
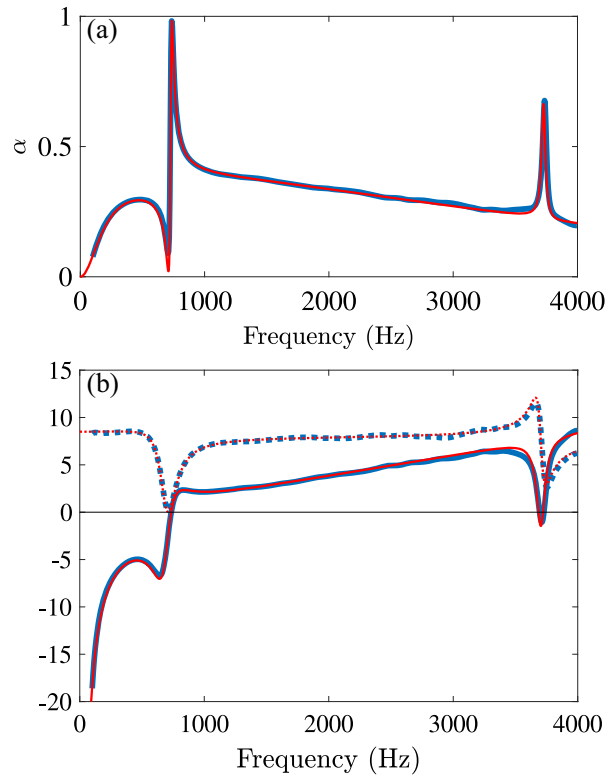

Figure 9. (a) Absorption coefficient and (b) impedance as functions of the frequency. The resistance are displayed by dashed lines and the reactance by solid lines. The experimental results (blue curves) are compared to the analytical results computed with the bi-articulated plate model (red curves).

downstream of the test section. A sinusoidal sweep ranging from $200 \mathrm{~Hz}$ to $4000 \mathrm{~Hz}$ with a step of $5 \mathrm{~Hz}$ is used.

The sound pressure in the duct is recorded by two sets of three flush-mounted microphones located upstream $\left(u_{i}\right)$ and downstream $\left(d_{i}\right)$ of the test section, where $i=1$ indicates the microphone located the closest to the test section. The positions of the microphones are $x_{u_{1}}-x_{u_{2}}=x_{d_{2}}-$ $x_{d_{1}}=30 \mathrm{~mm}, x_{u_{1}}-x_{u_{3}}=x_{d_{3}}-x_{d_{1}}=175 \mathrm{~mm}$, and both $u_{1}$ and $d_{1}$ are placed $113 \mathrm{~mm}$ away from the sample. All the microphones are calibrated relatively to $u_{1}$ in a separate cavity. At each frequency step of the sine sweep, the acoustic pressure on each microphone is calculated by averaging the pressure value over 400 cycles without flow, and over 1000 cycles with flow.

An overestimated determination of the incident and of the reflected waves on both sides of the sample is permitted by this mounting of six microphones. Then, the elements of the sample scattering matrix, namely the reflection and transmission coefficients $\left(r^{ \pm}, t^{ \pm}\right)$defined for incident plane waves coming from upstream $\left(r^{+}, t^{+}\right)$and downstream $\left(r^{-}, t^{-}\right)$of the sample, are computed. Two different acoustic states are needed to obtain these four coefficients. The first one consists in placing the compression chambers upstream of the test section, while they are located downstream for the second acoustic state [28].

As for the normal incidence case, this measuring system makes it possible to control the acoustic level of the incident wave. Several levels were tested but no non-linear effects were detected.

To predict the effect of the sample on propagation, two separate actions are required. The first one is to calculate the equivalent impedance of the sample. The second one 


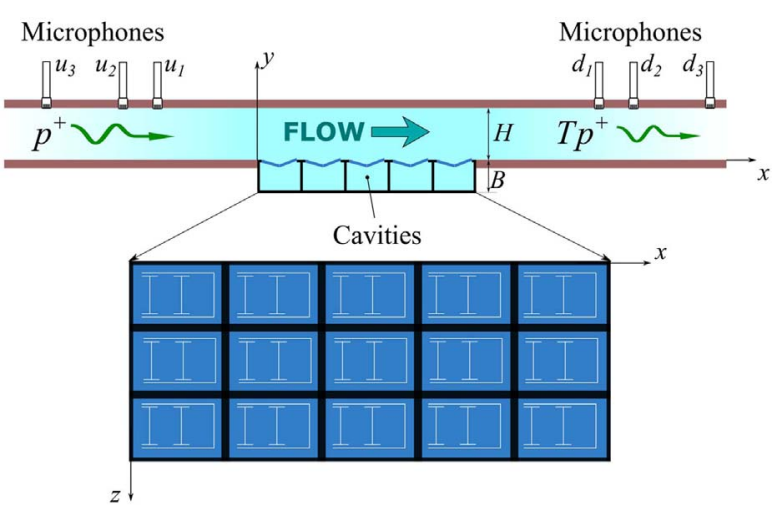

Figure 10. (Top) Schematic representation of the grazing incidence facility used to characterise the liner sample using two sets of three microphones. The duct has a rectangular cross section of height $H=4 \mathrm{~cm}$ and a rotating lobe blower is used to introduce flow. (Bottom) The composite plate has been glued over 15 cavities of section $15 \times 22 \mathrm{~mm}^{2}$ so that there is one cantilever beam per cavity. The height of the cavities is $B=30$ $\mathrm{mm}$.

is to predict the propagation in the duct in the presence of an acoustically treated wall.

The prediction of impedance is relatively easy since the impedances of the beam $Z_{b}$ and the slits $Z_{s}$ are identical to the case in normal incidence since the material and the geometry are the same. Similarly, since the cavity has the same depth $B$, the cavity impedance $Z_{c}$ is also unchanged. The only things that change in equation (5) are the incident sections $S_{i}=120 \times 50 / 15 \mathrm{~mm}^{2}$ and the cavity section $S_{c}=15 \times 22 \mathrm{~mm}^{2}$.

To predict the propagation with an impedance wall, a numerical simulation is carried out. To this end, a multimodal method is used to calculate the linearized twodimensional lossless problem. This method has already been described in detail elsewhere [28] and therefore only a few points are merely reported. The linear propagation of small perturbations can be described by the linearized Euler and continuity equations. The multimodal method is used and the perturbations are therefore expressed as a linear combination of the acoustic transverse modes. These modes and wave numbers are computed on uniform segments using a finite difference method by discretizing the equations in the transverse $y$-direction. Here, the modes must be calculated in the rigid duct and in the lined part wall. The scattering matrix of the sample is found by matching the modes at the discontinuities at each ends of the sample.

The comparison between the predicted and measured transmission and reflection coefficient is depicted in Figure 11. Due to reciprocity, without flow, the measured transmission coefficients in both directions are identical. Conversely, the reflection coefficients $r^{+}$and $r^{-}$differ slightly. This seems to indicate some inhomogeneity between the different beams which would not all react in the same way. This may for instance be due to the bonding of the plate to its support, which may not be exactly identical at every location. The comparison between predicted

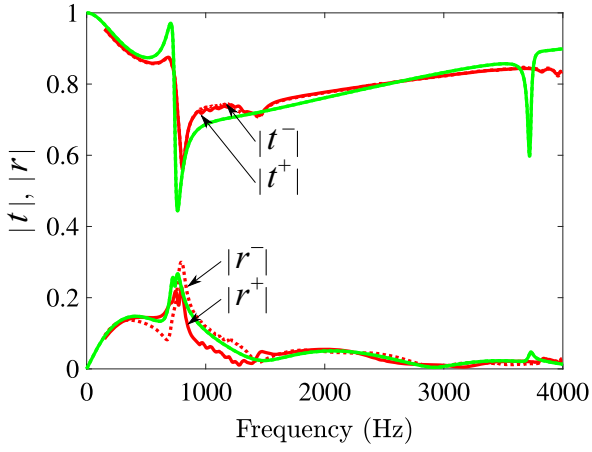

Figure 11. Transmission and reflection coefficients of the liner sample as a function of the frequency, without flow. The experimental results (red curves) are compared to the results provided by the multimodal method (green curves). For the latter, the boundary condition in the lined section is given by the bi-articulated plate model.

and measured values of transmission and reflection is relatively correct around the first resonance of the beam. It can be noted that the hypothesis that one can substitute a discrete set of cells, of fairly large size, with a distributed and homogeneous impedance can quickly find its limit when the frequency increases. Moreover, the implicit assumption that cells do not acoustically interfere with each other is also very questionable.

A striking effect is the disappearance of the second highfrequency peak (near $f_{2}=3730 \mathrm{~Hz}$ ). As $f=4000 \mathrm{~Hz}$ corresponds to the cut-off frequency of the second propagative mode in the rigid conduit that our setup does not allow to characterize, it was not possible to know if this second peak was rejected at higher frequency or if it simply disappeared. As mentioned above, for these frequencies the length of a cell is of the order of a quarter of the wavelength and the hypothesis of uniformity of impedance is no longer valid.

In spite of its approximations, the impedance homogenization model, as expressed from equation (8), gives good results at low frequencies and makes it possible to understand the main effects of treatments with vibrating articulated plates and micro slits on the propagation and reflection of the incident acoustic field.

\subsection{Effect of flow}

The implementation of the acoustic treatment in the wall of a duct allows studying the effect of a flow on its acoustic behavior. For this purpose, the duct installation is connected to a rotating lobe blower that can provide a mean velocity of up to $85 \mathrm{~m} / \mathrm{s}$. The flow velocity is measured at the center of the duct downstream of the test section by a Pitot tube connected to a differential pressure sensor. This measurement gives the maximum value of the flow velocity in the duct section. It is then multiplied by 0.8 in order to obtain the value of the average velocity and the Mach number M [29]. The measurements shown in Figure 12 were performed at a Mach number of 0.25 . 


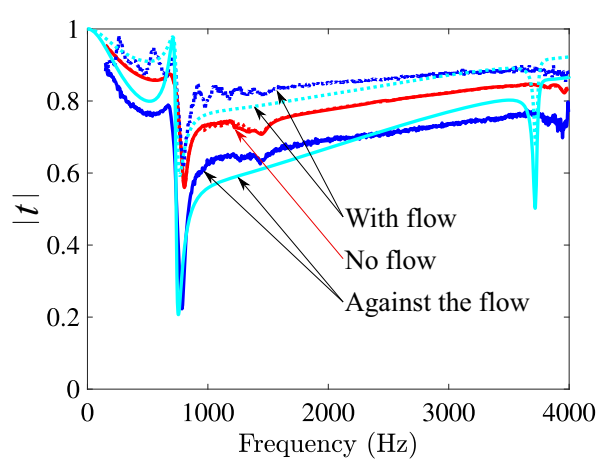

Figure 12. Transmission coefficients of the lined sample in presence of flow $(M=0.25)$. The experimental results (red curves without flow, blue curves with flow) are compared to the multimodal calculation (only the results with flow are shown, cyan curves).

The presence of an assumed uniform flow is also fairly easy to take into account in propagation modeling. To do this, it is necessary to add convection terms to the equations used. It is also necessary to modify the boundary condition that applies to the wall with impedance. Here we have used the classical condition of continuity of pressure and normal displacement, in general referred as the Ingard [30] - Myers [31] condition [32]. Finally, it is necessary to apply a special mode matching between the duct with impedance and the rigid duct that takes into account this Ingard-Myers condition [28].

The fairly good agreement between the experimental and the numerical results shows that the flow does not significantly change the value of the impedance of the material. The flow is therefore mainly introducing convection effects, both in the propagation itself and in the IngardMyers condition.

\section{Conclusion}

This paper shows that it is possible and interesting to use vibrating articulated plates combined with micro slits to obtain sound attenuation at low frequencies. Two behaviors exist in this type of structure. In the first one, the frequency is closed to the structure alone resonance. In the second one, only the structural mass is involved, the stiffness being provided by the cavity. In this second case, not studied in this paper [22], the frequency is higher for a given height of the cavity. A very simple model has been developed to describe the behavior of this type of structure both in normal and grazing incidence and fits well with the experiments. Compared to other types of acoustic surfaces [11], this type of structure does not seem to be very sensitive to the effects of sound level and grazing flow, with the latter being restricted to the convective effects on propagation.

Certainly, the operation of hinged plates with micro slits is rather narrow-band and fatigue problems can occur when using vibrating parts, but this device can tackle low frequency noise in parallel with other devices for other frequency ranges.

\section{Acknowledgments}

This research was supported by the European Union's Horizon 2020 research and innovation programme under the Marie Skłodowska-Curie grant agreement No. 722401 ("SmartAnswer") (https://www.h2020-smartanswer.eu/). The authors would like to thank Matthieu Giraud from Airbus Technocentre in Nantes for kindly supplying the composite plates.

\section{Conflict of interest}

The authors declare that they do not have any conflict of interst.

\section{References}

1. A. Guess: Calculation of perforated plate liner parameters from specified acoustic resistance and reactance. Journal of Sound and Vibration 40 (1975) 119-137.

2. D.-Y. Maa: Potential of microperforated panel absorber. The Journal of the Acoustical Society of America 104 (1998) 2861-2866.

3. M. Yang, S. Chen, C. Fu, P. Sheng: Optimal soundabsorbing structures. Materials Horizons 4 (2017) 673-680.

4. Y. Wu, M. Yang, P. Sheng: Perspective: Acoustic metamaterials in transition. Journal of Applied Physics 123 (2018) 090901.

5. B. Assouar, B. Liang, Y. Wu, Y. Li, J.-C. Cheng, Y. Jing: Acoustic metasurfaces. Nature Reviews Materials 1 (2018) 467-470.

6. X. Cai, Q. Guo, G. Hu, J. Yang: Ultrathin low-frequency sound absorbing panels based on coplanar spiral tubes or coplanar Helmholtz resonators. Applied Physics Letters 105 (2014) 121901.

7. C. Chen, Z. Du, G. Hu, J. Yang: A low-frequency sound absorbing material with subwavelength thickness. Applied Physics Letters 110 (2017) 221903.

8. Y. Wang, H. Zhao, H. Yang, J. Zhong, J. Wen: A space-coiled acoustic metamaterial with tunable low-frequency sound absorption. EPL (Europhysics Letters) 120 (2018) 54001.

9. S. Huang, X. Fang, X. Wang, B. Assouar, Q. Cheng, Y. Li: Acoustic perfect absorbers via Helmholtz resonators with embedded apertures. The Journal of the Acoustical Society of America 145 (2019) 254-262.

10. J.-P. Groby, W. Huang, A. Lardeau, Y. Aurégan: The use of slow waves to design simple sound absorbing materials. Journal of Applied Physics 117 (2015) 124903.

11. Y. Aurégan, M. Farooqui, J.-P. Groby: Low frequency sound attenuation in a flow duct using a thin slow sound material. The Journal of the Acoustical Society of America 139 (2016) EL149-EL153.

12. F. Simon: Long elastic open neck acoustic resonator for low frequency absorption. Journal of Sound and Vibration 421 (2018) 1-16.

13. W. Frommhold, H. Fuchs, S. Sheng, Acoustic performance of membrane absorbers, Journal of Sound and Vibration 170 (1994) 621-636.

14. L. Huang: Modal analysis of a drumlike silencer. The Journal of the Acoustical Society of America 112 (2002) 2014-2025.

15. G. Ma, M. Yang, S. Xiao, Z. Yang, P. Sheng: Acoustic metasurface with hybrid resonances. Nature Materials 13 (2014) 873. 
16. M. Yang, P. Sheng: Sound absorption structures: From porous media to acoustic metamaterials, Annual Review of Materials Research 47 (2017) 83-114.

17. Y. Aurégan: Ultra-thin low frequency perfect sound absorber with high ratio of active area. Applied Physics Letters 113 (2018) 201904.

18. X. Dai, Y. Aurégan: Flexural instability and sound amplification of a membrane-cavity configuration in shear flow. The Journal of the Acoustical Society of America 142 (2017) 1934-1942.

19. H.K. Fan, R.C. Leung, G.C. Lam, Y. Aurégan, X. Dai: Numerical coupling strategy for resolving in-duct elastic panel aeroacoustic/structural interaction. AIAA Journal 56 (2018) 5033-5040.

20. E. Martincic, A. Houdouin, S. Durand, N. Yaakoubi, E. Lefeuvre, Y. Aurégan: Acoustic absorber, acoustic wall and method for design and production (2019) US Patent 10,477,302.

21. M. Farooqui, Y. Aurégan: Compact beam liners for low frequency noise, in 2018 AIAA/CEAS Aeroacoustics Conference, 2018, 4101.

22. Y. Aurégan, M. Farooqui: In-parallel resonators to increase the absorption of subwavelength acoustic absorbers in the mid-frequency range. Scientific Reports 9 (2019) 1-6.

23. J.-F. Allard, N. Atalla: Propagation of sound in porous media: modelling sound absorbing materials 2e. John Wiley \& Sons, 2009.
24. M. Åbom: Measurement of the scattering-matrix of acoustical two-ports. Mechanical Systems and Signal Processing 5 (1991) 89-104.

25. M. Leroux, Y. Aurégan: Failures in the discrete models for flow duct with perforations: an experimental investigation, 265 (2003) 109-121.

26. M. D'Elia, T. Humbert, Y. Aurégan: Effect of flow on an array of helmholtz resonators: Is kevlar a "magic layer"? The Journal of the Acoustical Society of America 148 (2020) 3392-3396.

27. M.E. d'Elia, T. Humbert, Y. Auregan, J. Golliard: Optical measurements of the linear sound-flow interaction above a corrugated plate, in 25th AIAA/CEAS Aeroacoustics Conference, 2019, 2716.

28. Y. Aurégan, M. Leroux, V. Pagneux: Measurement of liner impedance with flow by an inverse method, in 10th AIAA/ CEAS Aeroacoustics Conference, 2004, 2838.

29. H. Schlichting: Boundary layer theory. 7th ed. McGraw-Hill, New York, 1979.

30. U. Ingard: Influence of fluid motion past a plane boundary on sound reflection, absorption, and transmission. The Journal of the Acoustical Society of America 31 (1959) 1035-1036.

31. M. Myers: On the acoustic boundary condition in the presence of flow. Journal of Sound and Vibration 71 (1980) 429-434.

32. Y. Renou, Y. Aurégan: Failure of the Ingard-Myers boundary condition for a lined duct: an experimental investigation, The Journal of the Acoustical Society of America 130 (2011) 52-60.

Cite this article as: D'Elia M.E. Humbert T. \& Aurégan Y. 2021. On articulated plates with micro-slits to tackle low-frequency noise. Acta Acustica, 5, 31. 